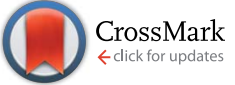

Cite this: RSC Adv., 2017, 7, 14422

\title{
Morphology effects on singlet oxygen production and bacterial photoinactivation efficiency by different silica-protoporphyrin IX nanocomposites $\uparrow$
}

\author{
G. Zampini, ${ }^{a b}$ O. Planas, ${ }^{b}$ F. Marmottini, ${ }^{a}$ O. Gulías, $^{b}$ M. Agut, ${ }^{b}$ S. Nonell ${ }^{\star b}$ \\ and L. Latterini*a
}

Different silica-protoporphyrin IX (PpIX) nanocomposites have been synthesized to evaluate the dependence of singlet oxygen production and bacterial inactivation efficiency on the morphology of the nanomaterials. Modulation of the synthetic procedure allowed obtaining silica nanoparticles with different porosity which were characterized by TEM and spectroscopic analysis after PpIX adsorption. Time-resolved phosphorescence measurements on the different nanoporous samples revealed that the porosity of the nanoparticles plays a pivotal role on the singlet oxygen production and release from the nanoparticles. Thus, apart from the expected decay of singlet oxygen outside the silica matrix, a second component has been observed for the porous materials, attributed to the decay of singlet oxygen inside the pores. The relative efficiency of singlet oxygen production resulted to be higher for the sample with the greatest pores volume. The capability of the nanocomposites to inactivate bacteria was tested in vitro on Staphylococcus aureus strain. Interestingly, the efficiency for singlet oxygen production of the nanocomposites and their bacterial inactivation efficiency followed a different trend, indicating that the relative position of the photosensitizer and the superficial properties of the particles affect the antibacterial activity of the overall system.

Received 18th January 2017

Accepted 21st February 2017

DOI: $10.1039 / c 7 r a 00784 a$

rsc.li/rsc-advances

\section{Introduction}

Antimicrobial photodynamic therapy (aPDT) is based on the use of a photosensitizer (PS), able to generate reactive oxygen species (ROS) through irradiation at specific wavelengths. ${ }^{1}$ It represents an effective treatment against antibiotic-resistant bacteria as ROS generated by PDT mechanism induce several damages on different levels of the cell, ${ }^{2,3}$ thus bacteria will not be easily able to develop resistance. ${ }^{4}$

Particular interest has been focused on singlet oxygen $\left({ }^{1} \mathrm{O}_{2}\right)$, which is conveniently produced by energy transfer between the excited triplet state of a PS and molecular oxygen, normally present in the surrounding environment. ${ }^{1}$

The increase in ${ }^{1} \mathrm{O}_{2}$ production efficiency is a crucial goal to achieve for an effective application of photodynamic systems. Hydrophobic natural PSs, such as protoporphyrin IX (PpIX), are potentially very promising but the extreme low solubility in physiological medium precludes their direct application in aPDT; ${ }^{5}$ indeed PpIX singlet excited states intersystem cross into

${ }^{a}$ Department of Chemistry, Biology and Biotechnology, University of Perugia, Via Elce di Sotto, 8, 06123 Perugia, Italy.E-mail: loredana.latterini@unipg.it

${ }^{b}$ Institut Quimic de Sarrià, Universitat Ramon Llull, Via Augusta 390, Barcelona 08017, Spain.E-mail: santi.nonell@iqs.url.edu

† Electronic supplementary information (ESI) available. See DOI: $10.1039 / \mathrm{c} 7 \mathrm{ra} 00784 \mathrm{a}$ triplet states with high quantum yields $\left(\Phi_{\mathrm{T}}=0.63 \pm 0.04\right),{ }^{6}$ and the long-lived triplet states are quenched efficiently by molecular oxygen to generate ${ }^{1} \mathrm{O}_{2}$ with a reported quantum yield values $\left(\Phi_{\Delta}\right)$

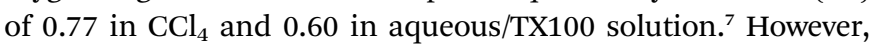
the formation of ground-state dimers and aggregates is responsible for the lowering of ${ }^{1} \mathrm{O}_{2}$ quantum yield, experimentally demonstrated by the reduction in $\Phi_{\Delta}$ value for increasing concentration of porphyrins in water, or with the increase in the ionic strength of the solution by the addition of $\mathrm{NaCl}^{8}$

In the last decades, several micro and nano-heterogeneous systems, in particular silica-based nanomaterials, have been studied and developed ${ }^{9}$ to overcome the solubility and the stability problems of the PS; ${ }^{10}$ the increasing interest for this kind of nanomaterials arises from the huge benefits related to the great stability of a silica structure, conjugated with the versatility of the particle surface. ${ }^{11}$ Indeed, very often they are used as a vehicle-system, ${ }^{12-15}$ firstly because the solid and inorganic structure of the matrix acts as a protective barrier against species that can destroy or limit the activity of PSs, ${ }^{16-19}$ and secondly the limitations due to the lability or the insolubility of these molecules may be overcome. ${ }^{18,20}$

The interactions between the photosensitizers and silica nanovectors have been recently investigated, with the aim to identify the most promising system for PDT. Different systems have been reported, wherein the non-covalent and covalent 
encapsulation of PpIX in both compact and mesoporous silica nanoparticles have been analyzed. ${ }^{21,22}$

Rossi and coworkers demonstrated that the covalent linkage of PpIX within the silica network allowed a significant improvement of the ability to produce singlet oxygen, obtaining a value of singlet oxygen quantum yield up to $0.9 .^{7}$

The synthetic approaches derived from the pioneering work of Prasad and Kopelman groups ${ }^{23,24}$ are based on the encapsulation of PpIX inside the core of silica nanoparticles, which were tested in PDT experiments both in vitro and in vivo. ${ }^{25,26}$ Vivero-Escoto et al. anchored PpIX to silica nanoparticles through the use of stimuli-responsive groups, in order to achieve the self-regulated delivery of the PS triggered by the biological system itself. ${ }^{27}$

Most of the strategies relay on the modulation of the interaction between PpIX and silica and on synthetic steps to tune such interactions. Limited studies have been presented to relate the morphology of silica nanoparticles to PDT performances; a detailed investigation on how the matrix nanostructures affect PpIX photophysical behavior and singlet oxygen production is concealing. However, the possibility to use the silica mesostructures to tune the chemical and photophysical behavior of PpIX represents an important simplification on the preparation procedures and possible scale-up. To achieve an improvement in smart nanomaterial development, it would be extremely important to connect the photophysical behavior to the photodynamic action of PS-loaded nanomaterials in physiological conditions. The anchoring on a solid nanosupport ${ }^{28,29}$ can inevitably modify not only the photophysics and the efficiency of a PS, but also the superficial properties of the materials, thus inducing eventual variations in the interactions with biological samples, such as bacteria.

Therefore, in this work, PpIX loaded on silica nanoparticles with different morphology have been analyzed through a systematic characterization of the ground, singlet and triplet excited states, evaluating how the morphology of the carrier affects the photophysical and photosensitizing pathways of the PS. Direct measurements of ${ }^{1} \mathrm{O}_{2}$ luminescence have been performed and the correlation to bacterial inactivation has been investigated to assess the influence of the physical-chemical properties of the nanocomposites to their antimicrobial behavior.

\section{Experimental section}

\section{Materials}

Protoporphyrin IX (PpIX, >97\%) was obtained in diacid form from Frontier Scientific and used without further purification. Tetraethylorthosilicate (TEOS, 98\%), (3-aminopropyl) triethoxysilane (APTES, $\geq 98 \%$ ), hexadecyltrimethylammonium bromide (CTAB , 95\%), hexadecyltrimethylammonium $p$-toluensulfonate (CTATos), triethanolamine $\left(\mathrm{TEAH}_{3},>98 \%\right)$, mesitylene (98\%), Pluronic F-127 $\left(\mathrm{EO}_{106} \mathrm{PO}_{60} \mathrm{EO}_{106}\right)$, hydrochloridric acid $(\mathrm{HCl}$, $37 \%)$ and ammonium hydroxide solution $\left(\mathrm{NH}_{4} \mathrm{OH}, 28.0-30.0 \%\right.$ $\mathrm{NH}_{3}$ basis), were all purchased from Sigma-Aldrich and used as received. Nanopure water $(\leq 15.0 \mathrm{M} \Omega$ ) from a Millipore Milli-Q gradient system, ethanol (96\%) and methanol (anhydrous, 99.8\%) from Sigma-Aldrich and $N, N$-dimethylformamide (DMF, $\geq 99 \%$ ) from Acros Organics were used as solvents. Staphylococcus aureus CECT 239 were obtained from the Spanish Type Culture Collection (CECT).

\section{Synthetic procedures}

Compact silica nanoparticles (CSNP). $56 \mathrm{~mL}$ of ethanol, $4 \mathrm{~mL}$ of water and $1.6 \mathrm{~mL}$ of ammonium hydroxide solution were mixed in a flask equipped with a magnetic bar. After few minutes, $2.4 \mathrm{~mL}$ of TEOS was quickly added to the mixture under vigorous magnetic stirring. After 24 hours the suspension was centrifuged $(3000 \mathrm{~g}, 30$ minutes) and the powder obtained was washed several times with ethanol and dried in air at room temperature. ${ }^{30}$

Mesoporous silica nanoparticles (MPSAm). $2.98 \mathrm{~g}$ of CTAB and $2.02 \mathrm{~g}$ of Pluronic $\mathrm{F}-127$ were added to $30 \mathrm{~mL}$ of $\mathrm{HCl}$ solution ( $\mathrm{pH}$ 0.5) under magnetic stirring; after 20 minutes $3.5 \mathrm{~g}$ of TEOS was added and the mixture was kept under magnetic stirring overnight. The gel formation occurred after the addition of $3.03 \mathrm{~g}$ of ammonium hydroxide solution $\left(\mathrm{NH}_{4} \mathrm{OH} 30 \%\right)$, and the system was aged for 24 hours at room temperature and then dried in air at $60{ }^{\circ} \mathrm{C}$ for 24 hours. Finally, solid samples were heated at $600{ }^{\circ} \mathrm{C}$ for 3 hours to completely remove the surfactant. ${ }^{31}$

"Stellate" mesoporous silica nanoparticles (MPSSt). A mixture of $762.8 \mathrm{mg}$ of hexadecyltrimethylammonium $p$-toluensulfonate, $97 \mu \mathrm{L}$ of triethanolamine and $41 \mathrm{~mL}$ of water was stirred for 1 hour at $80{ }^{\circ} \mathrm{C}$. Then $6.3 \mathrm{~mL}$ of TEOS was quickly added and the mixture was further stirred at $80{ }^{\circ} \mathrm{C}$ for 2 hours.

The as-synthesized MPSSt were filtered and washed several times with water and ethanol. The removal of the surfactant was obtained through solvent extraction in a boiling acidic methanol for 6 hours. The extracted particles were washed several times with ethanol and then dried in air. ${ }^{32}$

"Larger pore" mesoporous silica nanoparticles (MPSLP). A mixture of $620 \mathrm{mg}$ of hexadecyltrimethylammonium $p$-toluenesulfonate, $100 \mu \mathrm{L}$ of triethanolamine, $7.8 \mathrm{~mL}$ of mesitylene and $32.5 \mathrm{~mL}$ of water was stirred at $80^{\circ} \mathrm{C}$. After 1 hour, $7.8 \mathrm{~mL}$ of TEOS were quickly added and the mixture was stirred at $80{ }^{\circ} \mathrm{C}$ for 2 additional hours.

The MPSLP thus obtained were centrifuged at $3000 \mathrm{~g}$ for 15 minutes and washed several time with ethanol, followed by acidic extraction in methanol for at least 6 hours. The extracted particles were washed several time with ethanol and then dried in air.

APTES functionalization. CSNP, MPSAm, MPSSt and MPSLP colloids were functionalized with APTES in order to graft amino terminal groups on the surface of the particle.

In a typical synthesis procedure, a weighted amount of silica nanoparticles was suspended in ethanol and sonicated for 5 minutes to yield a final concentration of $5 \mathrm{mg} \mathrm{mL}{ }^{-1}$. Then APTES $\left(2 \mu \mathrm{L} \mathrm{mL}{ }^{-1}\right)$ was added and the mixture was stirred overnight at room temperature. The APTES-to-silica ratio was kept constant for all samples.

The amino-functionalized silica nanoparticles were obtained after centrifugation at $3000 \mathrm{~g}$ for 20 minutes, followed by several washes with ethanol. Finally, the solids were dried under air at room temperature. 
PpIX loading onto CSNP, MPSAm, MPSSt, MPSLP. PpIX anchorage was achieved by an acid-base reaction between the carboxylic acids of PpIX and the free amino groups on the silica surface. A fresh solution of PpIX in its acid form was prepared in DMF immediately before use. Then, a weighted amount of amino-functionalized silica nanoparticles was added to obtain a final concentration of $2 \mathrm{mg} \mathrm{mL}^{-1}$ for CSNP and $5 \mathrm{mg} \mathrm{mL}^{-1}$ for MPSAm, MPSSt and MPSLP. To obtain PpIX-CSNP, PpIXMPSAm, PpIX-MPSSt and PpIX-MPSLP, respectively. Each system was kept under magnetic stirring and stored in the dark for 24 hours.

Each silica suspension was then centrifuged at $3000 \mathrm{~g}$ for 20 minutes; the collected solid was washed twice with DMF and once with ethanol, and finally dried under air.

The concentration of PpIX on the silica surface was evaluated from the difference between the PpIX solution absorbance before (starting solution) and after (supernatant) the anchoring procedure (eqn $\mathrm{S} 1 \dagger$ ).

\section{Physicochemical characterization of the nanoparticles}

Size and morphology of all silica samples was investigated by a Philips 208 transmission electron microscope (TEM) operating at $80 \mathrm{kV}$. A small drop of the suspensions of aminofunctionalized nanoparticles in ethanol was deposited on a 300 square mesh copper grid pre-coated with a Formvar film and evaporated under air at room temperature. The particle size distribution was evaluated from several micrographs by means of the image analyser software ImageJ (Rasband, W.S., ImageJ, U. S. National Institutes of Health, Bethesda, Maryland, USA, http://imagej.nih.gov/ij/, 1997-2016). The zeta potential in aqueous suspensions was measured by a Nano-ZS Zetasizer (Malvern Instrument Ltd.). The samples were extensively sonicated prior to the measurements to ensure a complete dispersion of the nanoparticles.

Nitrogen adsorption-desorption isotherms at $77 \mathrm{~K}$ were determined using a computer controlled Micromeritics (Norcross, GA, USA) ASAP 2010 apparatus. Prior to adsorption measurements, samples were outgassed at room temperature overnight. The specific surface area was determined by the Brunauer, Emmett and Teller (B.E.T.) technique; ${ }^{33}$ mesopore volume was analysed by the BJH method. ${ }^{34}$

\section{Spectroscopic techniques}

All spectroscopic measurements were carried out in spectroscopic grade DMF using a nanoparticle concentration of $2 \mathrm{mg}$ $\mathrm{mL}^{-1}$ for all samples and after extensive sonication. Samples were sonicated before the measurements to ensure complete dispersion of the nanoparticles.

Absorption spectra were recorded on a Varian Cary $8454 \mathrm{UV} /$ Vis Diode Array and on a Varian Cary 6000i dual-beam spectrophotometers. Fluorescence emission spectra were recorded on a Jobin Yvon-Spex Fluoromax-4 spectrofluorometer. Timeresolved fluorescence emission decays were measured by use of PicoQuant Fluotime 200 time-correlated single photon counting system equipped with a red sensitive photomultiplier. Excitation was achieved using a $375 \mathrm{~nm}$ picosecond diode laser working at $10 \mathrm{MHz}$. Fluorescence lifetimes were determined using FluoFit 4.6.5 analysis software from PicoQuant. The specific ${ }^{1} \mathrm{O}_{2}$ near-infrared phosphorescence decays at $1270 \mathrm{~nm}$ were recorded by means of a customized PicoQuant Fluotime 200 system described in detail elsewhere. ${ }^{35}$ Briefly, a $532 \mathrm{~nm}$ diode-pumped Nd:YAG laser (FTSS355-Q, Crystal Laser) working at $1 \mathrm{KHz}$ repetition rate was used for excitation. Laser emission was filtered by a 1064 rugate notch filter (Edmund Optics) and an uncoated SKG-5 filter (CVI Laser Corportation) placed at the exit port of the laser to remove any residual component of its fundamental emission in the near-infrared. Sample luminescence was filtered by a 1100 long-pass filter (Edmund optics) and a narrow bandpass filter at $1270 \mathrm{~nm}$ (bk-1270-70-B, bk Interfernzoptik) to isolate the ${ }^{1} \mathrm{O}_{2}$ emission. Photons emitted from the sample were detected using a TE-cooled near-IR sensitive photomultiplier tube assembly (H9170-45, Hamamatsu Photonics) and counted with a multichannel scaler (NanoHarp 250, PicoQuant $\mathrm{GmbH}$ ). Time resolved-emission decays were fitted using non-linear regression function of Graph Pad Prism to recover the ${ }^{1} \mathrm{O}_{2}$ and triplet lifetimes $\left(\tau_{\Delta}\right.$ and $\tau_{\mathrm{T}}$, respectively). The quantum yield of ${ }^{1} \mathrm{O}_{2}$ production was derived from the amplitude of the signals.

Transient absorption experiments were carried out using a home-built nanosecond laser flash photolysis system. The second harmonic $(532 \mathrm{~nm})$ of a Continuum Surelite I-10 Nd:YAG laser ( $5 \mathrm{~ns}$ pulse width, $7.5 \mathrm{~mJ}$ per pulse) was directed to the sample and a $75 \mathrm{~W}$ short arc Xe lamp (USHIO) was used for probing the transient absorption in a right-angle geometry. Changes in the sample absorbance at different observation wavelengths were discriminated using a PTI 101 monochromator and detected using a Hamamatsu R928 photomultiplier. The signal was fed to a Lecroy WaveSurfer 454 oscilloscope for digitizing and averaging (typically 10 shot) and finally transferred to a PC computer for data storage and analysis. The energy of the laser pulsed was measured with a RJ 7610 energy meter from Laser Precision Corp. The system was controlled by a house-developed LKS software (LabView, National Instruments).

\section{Bacterial inactivation tests}

Microbial strains and growth conditions. Bacteria preinoculation was performed by adding the culture cells in sterile tryptic soy broth (TSB) and letting them grow overnight at $37^{\circ} \mathrm{C}$. Then stock inoculum suspension was prepared by diluting 100 $\mu \mathrm{L}$ of bacteria preinoculus in $10 \mathrm{~mL}$ of TSB. The suspension was kept in incubation at $37^{\circ} \mathrm{C}$ until the OD reached a value of 0.4 at $620 \mathrm{~nm}$ (equivalent to $c a \cdot 10^{8}$ colony-forming units), then centrifuged at $3000 \mathrm{~g}$ for 10 minutes, and the pellet obtained was washed once with sterilized PBS.

Photodynamic inactivation procedure. A weighted amount of dye-doped silica nanoparticles (PpIX-CSNP, PpIX-MPSAm, PpIX-MPSSt or PpIX-MPSLP) was added to the cell suspension in PBS in order to obtain a final concentration of $2 \mathrm{mg} \mathrm{mL}{ }^{-1}$. The as-prepared system was incubated at $37^{\circ} \mathrm{C}$ for 15 minutes, then $0.3 \mathrm{~mL}$ were placed in 96-well plates, and illuminated from the top by several blue-emitting lamps $(420 \mathrm{~nm}$, irradiance 10 
$\mathrm{mW} \mathrm{cm}^{-2}$, LZC-420, Luzchem) for different irradiation times. Serially 10 times-dilution were performed, and each well was seeded on tryptic soy agar in 4 replicas, and incubated at $37^{\circ} \mathrm{C}$ overnight. Colony-forming units (CFU) were counted to calculate the survival fractions. A similar protocol was applied for the dark control, with the exception of the irradiation procedure.

\section{Results and discussion}

\section{Silica nanoparticles morphology and superficial properties}

Fig. 1 shows the TEM images of the amino-functionalized nanostructures. Rapid inspection of the different contrast TEM images reveals that the prepared nanoparticles present four different types of morphologies: compact regular silica nanoparticles (CSNP, A), amorphous mesoporous silica nanoparticles (MPSAm, B), stellate mesoporous silica nanoparticles (MPSSt, C) and large-pore mesoporous silica nanoparticles (MPSLP, D). The mean diameter obtained for CSNP, MPSSt and MPSLP (see ESI, Fig. S1-S3 $\uparrow$ ), was respectively $117 \mathrm{~nm}(\sigma=7.1$ $\mathrm{nm}), 83 \mathrm{~nm}(\sigma=8.8 \mathrm{~nm})$ and $94 \mathrm{~nm}(\sigma=8.8 \mathrm{~nm})$. It was not possible to accurately evaluate the size distribution for MPSAm due to the irregular nature of its structure, however nanostructures having tens of nm dimensions can be observed.

The control of nanoparticle's dimensions was achieved by the tight regulation of reactant's concentrations and synthetic conditions; the differential morphology of the silica nanoparticles is the result of templated synthetic strategies used for the sample preparation. In particular, CSNP were synthesized following the Stöber procedure with minor changes in the relative quantity of water, ethanol and ammonia, to achieve a good monodisperse size distribution for CSNP sample. ${ }^{30}$ The different pore shapes and morphologies for samples B, C and D (Fig. 1), were achieved by using different templating agents. Pluronic F127 yielded highly curved cage-like pores ${ }^{36}$ with small diameter, ${ }^{31}$ as in the case of MPSAm. Instead, the cationic surfactant cetyltrimethylammonium $\left(\mathrm{CTA}^{+}\right)$associated to
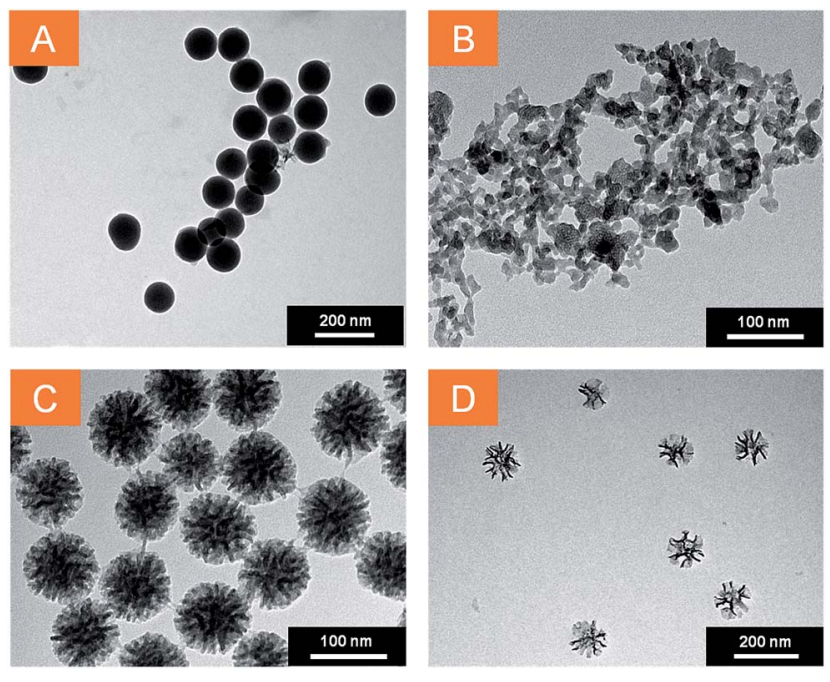

Fig. 1 TEM images of silica nanoparticles. (A) CSNP scale bar: $200 \mathrm{~nm}$, (B) MPSAm scale bar: $100 \mathrm{~nm}$, (C) MPSSt scale bar: $100 \mathrm{~nm}$, (D) MPSLP scale bar: $200 \mathrm{~nm}$. tosilate $\left(\operatorname{Tos}^{-}\right)$, a counterion with strong affinity to the polar head of the surfactant, ${ }^{32}$ led to the formation of a star-shaped ("stellate") structure for MPSSt. Finally, the addition of mesitylene, a swelling agent, to the reaction mixture caused an increase in the dimensions of the pores ${ }^{37}$ without modifying their star shape (MPSLP). The achieved morphologies were not altered by amino-functionalization.

The surface area data of the nanosystems as well as zeta potential values in aqueous suspensions are summarized in Table S1.†

The adsorption and desorption isotherms of all the silica samples presented similar profiles, reflecting a mesoporous structure for all the samples; BET analysis of the data evidences that the surface area increases in the order CSNP, MPSAm, MPSSt and MPSLP, in agreement with the TEM findings. The evaluation of mesopore volume and mesopore size distribution was performed by BJH analysis of the adsorption and desorption data. The mesopore volume calculated from adsorption data for pores of dimensions between $20 \AA$ and $400 \AA$ is reported in Table S1 and in Fig. S5 $\uparrow$ the mesopore size distribution curves are showed. It is of interest to note that in the case of CSNP sample the calculated mesopore volume is $0.13 \mathrm{~cm}^{3} \mathrm{~g}^{-1}$ while the values obtained for others samples are included in the range 0.44-0.52 $\mathrm{cm}^{3} \mathrm{~g}^{-1}$. The pore size distribution curves put in evidence that, differently from the other samples, a wide maximum occur around a pore diameter of $100 \AA$ in the case of MPSSt sample.

At the same time, a clear trend of the final zeta-potential values going from CSNP to MPSLP can be observed, which correlates with the surface area and pore-structuring of the nanomaterials. As porosity is increased, the zeta-potential diminishes. It is known that bare silica nanoparticles are generally negatively charged in water because of the presence of partly ionized silanol groups, whereas surface functionalization with terminal amino groups increases the value of the zeta potential..$^{38,39}$ The results suggest that the pore size plays a fundamental role on the zeta potential values. This observation can be rationalized considering that APTES grafting has been carried out keeping constant the ratio of silica and APTES concentrations for all samples. The larger are the pores, the higher is the exposed surface (inner and outer the pores). Moreover, the samples bearing larger pores have a higher surface concentration of silanol moieties, resulting in lower zeta potentials. As a result, the surface of the nanoparticles with larger structures is on average, less covered by positively charged amino groups in the nanoparticles with larger pores, yielding to a more negative zeta potential value. Accordingly, for CSNP, in which no pores are present, the most positive zeta potential value is obtained.

\section{Silica-PpIX conjugates}

PpIX was electrostatically attached onto the different aminofunctionalized silica nanostructures suspended in DMF by acidbase reaction of its carboxylic acid groups with the nanoparticle amino groups, and the samples PpIX-CSNP, PpIX-MPSAm, PpIXMPSSt and PpIX-MPSLP were obtained. The concentration of PpIX ( $\mathrm{nmol}_{\mathrm{PpIX}} / \mathrm{mg}_{\text {silica }}$ ) on the different silica samples was quantified through spectrophotometric measurements and is given in 
Table S1. $\dagger$ The concentration is the highest for CSNPs, and roughly equal for all mesoporous nanomaterials, independent of the pore size. Conjugation of PpIX did not modify the zeta potentials to a great extent (Table $\mathrm{S} 1 \dagger$ ).

\section{Photophysical properties of silica-PpIX conjugates}

To evaluate the effect of the silica matrix on the photophysical behavior of PpIX, each sample was re-suspended in DMF and their absorption and fluorescence spectra were recorded. The scattering corrected absorption spectra of PpIX-loaded nanoconstructs are shown in Fig. 2a. For all samples, the classical shape of PpIX absorption is observed, with an intense Soret band centered at $406 \mathrm{~nm}$ and several $\mathrm{Q}$ bands in the visible range. However, an additional band at $675 \mathrm{~nm}$, attributed to aggregated species ${ }^{\mathbf{4 0 - 4 2}}$ can be detected for all samples, albeit with different intensities.

Fluorescence spectra (Fig. 2b) likewise show the typical shape of PpIX but a new band at $680 \mathrm{~nm}$ is observed, whose relative intensity varies from sample to sample. Porphyrins have a strong tendency to aggregate and dimers are known to show red-shifted fluorescence relative to monomers, while higherorder aggregates are non-fluorescent. ${ }^{41}$ The observed spectra are consistent with the presence of both fluorescent dimers and non-fluorescent higher-order aggregates. Thus, PpIX-MPSLP displays the highest fluorescence intensity while PpIX-CNSP
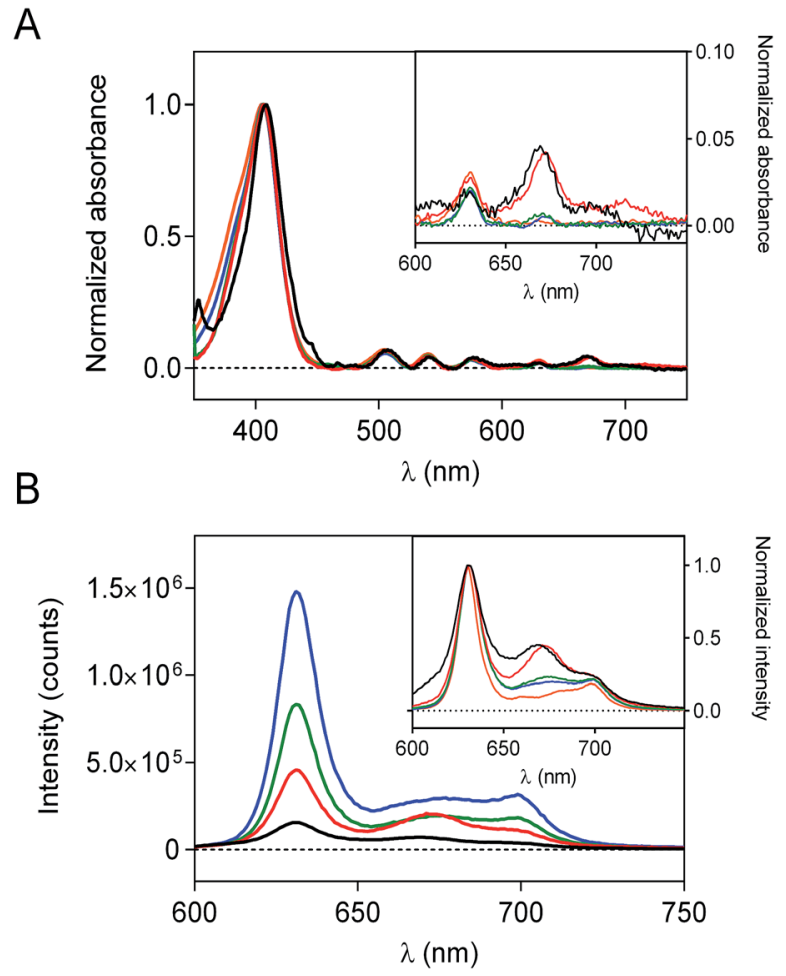

Fig. 2 (A) Normalized absorption spectra of different silica nanoconjugate suspensions (black line PpIX-CSNP, red line PpIX-MPSAm, green line PpIX-MPSSt and blue line PpIX-MPSLP) in concentration of $2 \mathrm{mg} \mathrm{mL}^{-1}$ in DMF. Inset: magnification between $650-700 \mathrm{~nm}$. (B) Overlaid fluorescence emission spectra $\left(\lambda_{\text {exc }}=532 \mathrm{~nm}\right)$; normalized fluorescence emission spectra, including PpIX emission in DMF, is shown as an inset for comparison purposes.
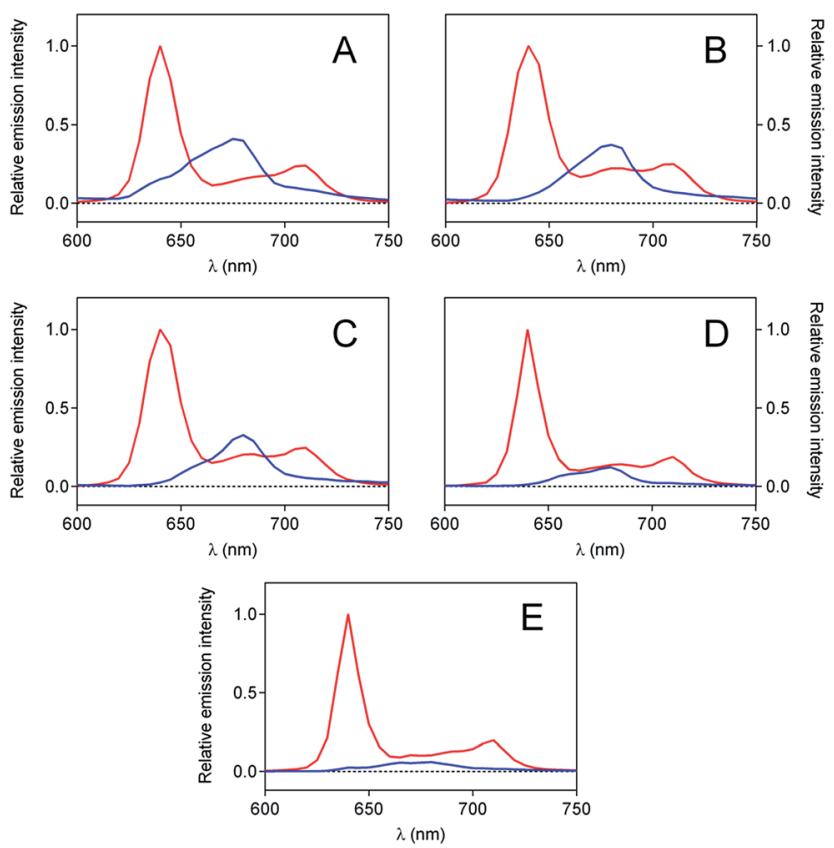

Fig. 3 Time-resolved fluorescence spectra of (A) PpIX-CSNP, (B) PpIX-MPSAm, (C) PpIX-MPSSt, (D) PpIX-MPSLP, and (E) PpIX following the excitation at $532 \mathrm{~nm}$. $14 \mathrm{~ns}$ component is indicated with a red line and 5 ns in blue.

shows the lowest, despite their respective PpIX loading show the opposite trend (Table S1 $\dagger$ ). This indicates that PpIX is most aggregated in CNSP, consistent with its lowest specific surface.

The aggregation phenomenon was further confirmed by time-resolved fluorescence spectroscopy (Fig. 3). For each suspension, two contributions to the fluorescence decay were

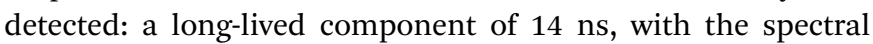
features of monomeric PpIX in DMF, ${ }^{43}$ and a shorter-lived component of about $5 \mathrm{~ns}$, attributed to the fluorescent dimers. ${ }^{\mathbf{4 4}}$

Further analysis was conducted to characterize the photophysical behavior of PpIX triplet state, both conjugated and unconjugated to silica, in air and in argon-saturated solution (Fig. S6 and Table S2†). All transient absorption signals could be satisfactorily fitted with a monoexponential decay function. In air-saturated samples, a triplet lifetime of about $0.4 \mu$ s was found for all the samples. However, when oxygen was removed from the suspensions through extensive argon bubbling, large differences were observed between all samples with a clear trend: the larger the pore, the longer the triplet lifetime becomes, suggesting that porous silica nanoparticles protect PpIX from self-quenching. ${ }^{45}$

\section{Singlet oxygen measurements}

Kinetic studies. Time-resolved phosphorescence signals at $1270 \mathrm{~nm}$ for both free PpIX and PpIX-CSNP conjugates in DMF (Fig. 4) could be fitted using the classic rise and decay biexponential model (eqn (1)), ${ }^{35}$ where $\tau_{\Delta}$ is the singlet oxygen lifetime and $\tau_{\mathrm{T}}$ the triplet lifetime.

$$
S_{t}=S_{0} \times \frac{\tau_{\Delta}}{\tau_{\Delta}-\tau_{\mathrm{T}}} \times\left(\mathrm{e}^{-t / \tau_{\Delta}}-\mathrm{e}^{-t / \tau_{\mathrm{T}}}\right)
$$




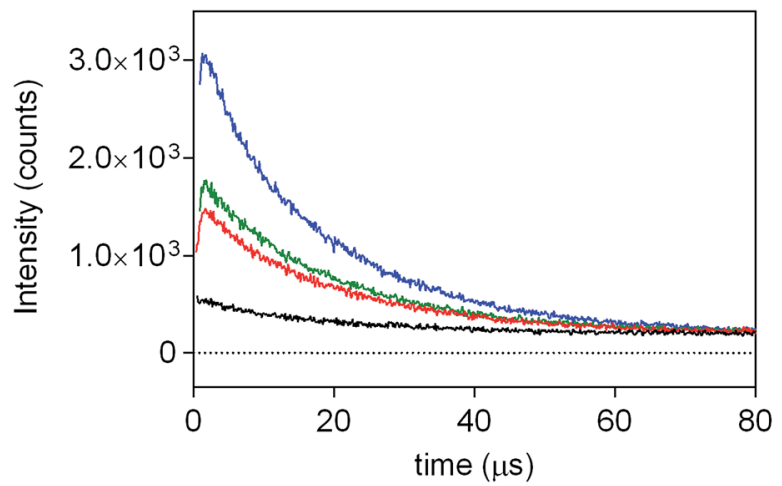

Fig. 4 Time-resolved ${ }^{1} \mathrm{O}_{2}$ phosphorescence spectra of PpIX-CSNP (black line), PpIX-MPSAm (red line), PpIX-MPSSt (green line) and PpIXMPSLP (blue line) acquired at $1270 \mathrm{~nm}$ with an excitation wavelength of $532 \mathrm{~nm}$.

In contrast, singlet oxygen luminescence signals from mesoporous nanomaterials required the introduction of an additional decay component to adequately reproduce the experimental traces (eqn (2)).

$$
\begin{aligned}
S_{t}= & S_{0,1} \times \frac{\tau_{\Delta 1}}{\tau_{\Delta 1}-\tau_{\mathrm{T} 1}} \times \mathrm{e}^{-t / \tau_{\Delta 1}}+S_{0,2} \times \frac{\tau_{\Delta 2}}{\tau_{\Delta 2}-\tau_{\mathrm{T} 2}} \\
& \times \mathrm{e}^{-t / \tau_{\Delta 2}}-\left(S_{0,1} \times \frac{\tau_{\Delta 1}}{\tau_{\Delta 1}-\tau_{\mathrm{T}}}+S_{0,2} \times \frac{\tau_{\Delta 2}}{\tau_{\Delta 2}-\tau_{\mathrm{T}}}\right) \times \mathrm{e}^{-t / \tau_{\mathrm{T}}}
\end{aligned}
$$

Results from fittings are summarized in Table 1. PpIX and PpIX-CSNP showed a growth lifetime of 0.4 and $0.5 \mu$ s and a decay lifetime of 22 and $20 \mu \mathrm{s}$, respectively. These lifetimes match those observed for molecular PS in DMF. ${ }^{46}$ In mesoporous nanomaterials, a similar rise lifetime was observed (0.3$0.5 \mu \mathrm{s}$ ) along with two decay components of $3 \mu \mathrm{s}$ and 19-20 $\mu \mathrm{s}$. In order to assign the observed lifetimes, phosphorescence decays were also recorded under oxygen-saturated conditions. A clear shortening of the rise component was observed while the decay components remained almost unaffected (Fig. S7 and Table S3 $\uparrow$ ). Taken together with the results of the transient absorption experiments, these data indicate that the rise component corresponds to the formation of ${ }^{1} \mathrm{O}_{2}$ from the triplet state of PpIX and the decay components to the decay of ${ }^{1} \mathrm{O}_{2}$.

In order to explain these results, it is worth recalling that while in compact nanoparticles only the external surface is available for anchoring the PS, the porosity of the mesoporous nanoparticles may enable the localization of PpIX both on the external surface and inside the silica pores. It can therefore be proposed that two populations of ${ }^{1} \mathrm{O}_{2}$ might exist; one formed at the silica-solvent interface and able to escape and relax in the outer bulk solvent and another that decays inside the silica pores. The external ${ }^{1} \mathrm{O}_{2}$ population decays therefore with a lifetime of $20 \mu \mathrm{s}$, as in the case of PpIX-CSNP. The inner- ${ }^{1} \mathrm{O}_{2}$ population, trapped in the silica structures, shows a much faster decay, probably due to a combination of three different effects: (i) an increase in the collision frequency of ${ }^{1} \mathrm{O}_{2}$ with the walls of the pores, ${ }^{47}$ (ii) quenching of ${ }^{1} \mathrm{O}_{2}$ by free amino groups deriving
Table 1 Fitting parameters obtained from the analysis of timeresolved phosphorescence signals at $1270 \mathrm{~nm}$ for different silica-PpIX suspensions in air-saturated conditions at $2 \mathrm{mg} \mathrm{mL}^{-1}$ of silica

\begin{tabular}{llllll}
\hline Conjugate & $S_{0,1} / \mathrm{cnts}$ & $S_{0,2} / \mathrm{cnts}$ & $\tau_{\mathrm{T}} / \mu \mathrm{s}$ & $\tau_{\Delta 1} / \mu \mathrm{s}$ & $\tau_{\Delta 2} / \mu \mathrm{s}$ \\
\hline PpIX & N/A & - & 0.4 & 22 & - \\
PpIX-CSNP & 341 & - & 0.5 & 19.9 & - \\
PpIX-MPSAm & $1291(95 \%)$ & $71(5 \%)$ & 0.4 & 19.5 & 3.0 \\
PpIX-MPSSt & $1515(92 \%)$ & $125(8 \%)$ & 0.3 & 19.5 & 3.0 \\
PpIX-MPSLP & $2584(76 \%)$ & $792(24 \%)$ & 0.5 & 19.2 & 3.1
\end{tabular}

from APTES functionalization, ${ }^{48,49}$ or (iii) quenching by $\mathrm{OH}$ groups of the surface silanols and of strongly hydrogen-bonded water molecules remaining from the nanoparticle preparation procedure. $^{50}$

This interpretation was confirmed by a second series of experiments in which PPIX-MPSLP was re-suspended in $\mathrm{D}_{2} \mathrm{O}$. It is well known that OD groups, produced by isotopic exchange of the trapped water molecules and surface silanols with $\mathrm{D}_{2} \mathrm{O}$, are far worse quenchers of ${ }^{1} \mathrm{O}_{2}$ than $\mathrm{OH}$ groups. ${ }^{51}$ One would therefore expect that the ${ }^{1} \mathrm{O}_{2}$ lifetime inside the pores would now be closer to that in the external bulk solvent. Consistent with this expectation, the phosphorescence decay could be satisfactorily fitted by a biexponential model with a $3 \mu$ s rise time and $53 \mu$ s for the single decay component (Fig. S8 $\dagger$ ). One can notice that the determined ${ }^{1} \mathrm{O}_{2}$ decay time value $(53 \mu \mathrm{s})$ is slightly shorter than the reported lifetime of ${ }^{1} \mathrm{O}_{2}$ in $\mathrm{D}_{2} \mathrm{O}$, (67 $\mu \mathrm{s}),{ }^{52}$ indicating that silica still exerts some quenching effect.

Singlet oxygen production efficiency. From the fitted initial amplitude of the ${ }^{1} \mathrm{O}_{2}$ signal $\left(S_{0}\right)$, it is possible to estimate the relative singlet oxygen production quantum yield $\left(\Phi_{\Delta}\right)$, which indicates the ability of a system to sensitize ${ }^{1} \mathrm{O}_{2}$ with respect to a reference. Given that PpIX-CSNP was the sample with the lowest ${ }^{1} \mathrm{O}_{2}$ production, it has been taken as a reference. For the mesoporous samples, $\Phi_{\Delta}$ was evaluated according to eqn (3) where $k_{\mathrm{R}, \mathrm{DMF}}$ and $k_{\mathrm{R}, \mathrm{H}_{2} \mathrm{O}}$ are the radiative rate constants for ${ }^{1} \mathrm{O}_{2}$ in DMF and $\mathrm{H}_{2} \mathrm{O}\left(0.63 \mathrm{~s}^{-1}\right.$ and $0.209 \mathrm{~s}^{-1}$, respectively). ${ }^{51}$ This correction is necessary to account for the fact that the probability of radiative decay is higher in DMF than in $\mathrm{H}_{2} \mathrm{O}$.

$$
\text { Relative } \Phi_{\Delta}=\frac{\Phi_{\Delta}^{\text {mesoporous }}}{\Phi_{\Delta}^{\mathrm{CSNP}}}=\frac{S_{0,1}^{\text {mesoporous }}+\frac{k_{\mathrm{R}, \mathrm{DMF}}}{k_{\mathrm{R}, \mathrm{H}_{2} \mathrm{O}}} S_{0,2}^{\text {mesoporous }}}{S_{0}^{\mathrm{CSNP}}}
$$

As shown in Table 2, the mesoporous materials have a higher efficiency in the photosensitization of ${ }^{1} \mathrm{O}_{2}$. In particular, PpIXMPSAm, PpIX-MPSSt and PpIX-MPSLP display a relative efficiency about 4.4, 5.5 and 14.6 times higher than PpIX-CSNP, respectively, with PpIX-MPSLP presenting the highest value. However, in the mesoporous materials, the two populations of ${ }^{1} \mathrm{O}_{2}$ molecules, kinetically detected (Table 1), might show different effectiveness to photoinactivate bacteria. The relative contribution of the two populations can be estimated from the amplitude of the components corrected for the radiative rate constants as detailed in eqn (4) and is also given in Table 2. 
Table 2 Singlet oxygen production efficiency of silica nanomaterials related to PpIX-CSNP. All samples were measured using the same silica concentration $\left(2 \mathrm{mg} \mathrm{mL}^{-1}\right)$

\begin{tabular}{lll}
\hline Sample & $\begin{array}{l}\text { Loading } \mathrm{nmol}_{\mathrm{PpIX}} / \\
\mathrm{mg}_{\text {silica }}\end{array}$ & $\begin{array}{l}\text { Relative }{ }^{1} \mathrm{O}_{2} \text { efficiency } \\
(\% \text { outer population) }\end{array}$ \\
\hline PpIX-CSNP & 1.01 & $1(100 \%)$ \\
PPIX-MPSAm & 0.74 & $4.4(86 \%)$ \\
PpIX-MPSSt & 0.60 & $5.5(81 \%)$ \\
PpIX-MPSLP & 0.64 & $14.6(52 \%)$
\end{tabular}

$$
\text { Outer }{ }^{1} \mathrm{O}_{2}(\%)=\frac{S_{0,1}^{\text {mesoporous }}}{S_{0,1}^{\text {mesoporous }}+\frac{k_{\mathrm{R}, \mathrm{DMF}}}{k_{\mathrm{R}, \mathrm{H}_{2} \mathrm{O}}} S_{0,2}^{\text {mesoporous }}} \times 100
$$

\section{Bacterial inactivation}

The photoinduced antibacterial property of PpIX-doped silica nanoparticles was tested to correlate this activity with the morphology and ${ }^{1} \mathrm{O}_{2}$ production capacity. Staphylococcus aureus was chosen as a model Gram positive bacteria, and the concentration of all silica samples was kept constant (see Table S4 in the $\mathrm{ESI} \dagger$ ) to properly compare the efficiency in bacterial killing.

As expected, neither bare silica nanoparticles nor any of the PpIX-silica conjugates showed any remarkable dark toxicity towards S. aureus (Fig. 5). Moreover, bare silica nanoparticles, with no PpIX adsorbed, were non-toxic even at the highest light doses tested $\left(12 \mathrm{~J} \mathrm{~cm}^{-2}\right)$.

The efficacy of bacterial photoinactivation varied dramatically for the different PpIX-loaded samples, highlighting the importance of the nanoparticle structure. PpIX-MPSAm, which has an undefined geometry and irregular structure, does not develop any photo-induced antibacterial activities; indeed for this sample a decrease in bacterial population comparable to the one measured in the dark, has been observed. Thus, this material was not considered worth of further scrutiny.

Among the samples with well-defined nanostructures, the highest reduction in the survival of $S$. aureus was achieved upon irradiation of PpIX-CSNP; the result can be quite surprising

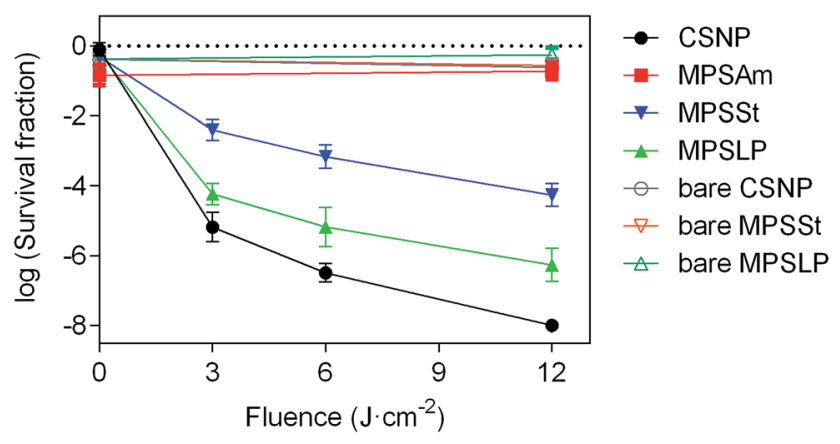

Fig. 5 Survival curves of $S$. aureus incubated with different silica nanoconjugates; the closed symbols are used to indicate the systems with PpIX (in solution or anchored onto silica surface), whereas the open symbols are the control experiments (bare silica nanoparticles with no photosensitizer adsorbed). The irradiation was performed at $420 \mathrm{~nm}$. since this nanomaterial shows the lowest ${ }^{1} \mathrm{O}_{2}$ production efficacy (Table 2). A possible explanation for such apparent contradiction is that its positive zeta potential enables the closest proximity to the bacteria, thereby facilitating the delivery of ${ }^{1} \mathrm{O}_{2}$ and/or PpIX to the bacteria. ${ }^{53-55}$

Among the mesoporous nanomaterials, those with large pores (PpIX-MPSLP) showed higher antibacterial efficacy than those with small pores (PpIX-MPSSt), which correlates with the respective ${ }^{1} \mathrm{O}_{2}$ quantum yields and the extent of PpIX aggregation. One could probably expect that if their zeta potentials had been as positive as those of CSNP, their antibacterial efficacy would be higher.

Therefore, the effort to direct the synthesis toward the construction of nanomaterials with definite geometry is crucial to maximize the exploitation of their properties. These results underline that the increase in $\Phi_{\Delta}$ is not necessarily the ultimately goal for achieving maximum efficiency in bacterial photoinactivation, factors such as zeta potential and morphology appear at least as crucial. Therefore, for a complete evaluation of new systems, photophysical studies should be correlated with the nanoparticle structure using physicochemical and biological assays to ascertain the effective improvement of the overall efficiency.

\section{Conclusions}

In this work, deeper insight on the photophysical behavior, singlet oxygen production efficiency and antimicrobial activity of PpIX-doped silica nanoparticles has been achieved through correlation of the different silica morphology with the optical and photophysical properties of PpIX-silica conjugate.

From our results, it can be derived that the silica nanoparticles' morphology and PpIX localization in the inorganic matrix cause major changes in the photophysical behavior of PpIX, in the photosensitization of ${ }^{1} \mathrm{O}_{2}$ and in the bacterial photoinactivation efficiency.

\section{Acknowledgements}

Financial support for this research was obtained from the Spanish Ministerio de Economía y Competitividad (Grant No. CTQ2013-48767-C3-1-R) and the University of Perugia. G.Z. thanks the support of the University of Perugia for the Erasmus fellowship. O.P. thanks the European Social Funds and the SUR del DEC de la Generalitat de Catalunya for his predoctoral fellowship (grant No. 2016 FI_B2_00100).

\section{Notes and references}

1 D. R. Kearns, Chem. Rev., 1971, 71, 395-427.

2 H. Pelicano, D. Carney and P. Huang, Drug Resist. Updates, 2004, 7, 97-110.

3 R. Dosselli, R. Ruiz-González, F. Moret, V. Agnolon, C. Compagnin, M. Mognato, V. Sella, M. Agut, S. Nonell, M. Gobbo and E. Reddi, J. Med. Chem., 2014, 57, 1403-1415. 
4 F. Giuliani, M. Martinelli, A. Cocchi, D. Arbia, L. Fantetti and G. Roncucci, Antimicrob. Agents Chemother., 2010, 54, 637642.

5 H. Ding, B. D. Sumer, C. W. Kessinger, Y. Dong, G. Huang, D. A. Boothman and J. Gao, J. Controlled Release, 2011, 151, 271-277.

6 C. B. Nielsen, J. S. Forster, P. R. Ogilby and S. B. Nielsen, J. Phys. Chem. A, 2005, 109, 3875-3879.

7 L. M. Rossi, P. R. Silva, L. L. R. Vono, a U. Fernandes, D. B. Tada and M. S. Baptista, Langmuir, 2008, 24, 12534-12538.

8 D. G. Fresnadillo and S. Lacombe, in Singlet Oxygen: Applications in Biosciences and Nanosciences, 2016, vol. 1, pp. 105-143.

9 J. L. Vivero-Escoto, in Photodynamic Therapy: Fundamentals, Applications and Health Outcomes, 2015, pp. 1-35.

10 Y. Hsia, M. Sivasubramanian, N.-T. Chen and L.-W. Lo, in Nanomaterials for Tumor Targeting Theranostics: A Proactive Clinical Perspective, 2016, pp. 143-176.

11 V. Ambrogi, A. Donnadio, D. Pietrella, L. Latterini, F. A. Proietti, F. Marmottini, G. Padeletti, S. Kaciulis, S. Giovagnoli and M. Ricci, J. Mater. Chem. B, 2014, 2, 6054.

12 V. Ambrogi, L. Perioli, C. Pagano, L. Latterini, F. Marmottini, M. Ricci and C. Rossi, Microporous Mesoporous Mater., 2012, 147, 343-349.

13 L. Latterini and L. Tarpani, J. Phys. Chem. C, 2011, 115, 21098-21104.

14 Z. Chu, S. Zhang, C. Yin, G. Lin and Q. Li, Biomater. Sci., 2014, 2, 827-832.

$15 \mathrm{~J}$. Vivero-Escoto and M. Elnagheeb, Nanomaterials, 2015, 5, 2302-2316.

16 M. Manzano, V. Aina, C. O. Areán, F. Balas, V. Cauda, M. Colilla, M. R. Delgado and M. Vallet-Regí, Chem. Eng. J., 2008, 137, 30-37.

17 I. I. Slowing, B. G. Trewyn, S. Giri and V. S. Y. Lin, Adv. Funct. Mater., 2007, 17, 1225-1236.

18 I. I. Slowing, J. L. Vivero-Escoto, C.-W. Wu and V. S.-Y. Lin, Adv. Drug Delivery Rev., 2008, 60, 1278-1288.

19 C. Barbé, J. Bartlett, L. Kong, K. Finnie, H. Q. Lin, M. Larkin, S. Calleja, A. Bush and G. Calleja, Adv. Mater., 2004, 16, 19591966.

20 R. Singh and J. W. Lillard, Exp. Mol. Pathol., 2009, 86, 215223.

21 F. Figueira, J. A. S. Cavaleiro and J. P. C. Tomé, J. Porphyrins Phthalocyanines, 2011, 15, 517-533.

22 P. Couleaud, V. Morosini, C. Frochot, S. Richeter, L. Raehma and J.-O. Durand, Nanoscale, 2010, 2, 1083-1095.

23 I. Roy, T. Y. Ohulchanskyy, H. E. Pudavar, E. J. Bergey, A. R. Oseroff, J. Morgan, T. J. Dougherty and P. N. Prasad, J. Am. Chem. Soc., 2003, 125, 7860-7865.

24 F. Yan and R. Kopelman, Photochem. Photobiol., 2003, 78, 587-591.

25 V. Simon, C. Devaux, A. Darmon, T. Donnet, E. Thiénot, M. Germain, J. Honnorat, A. Duval, A. Pottier, E. Borghi, L. Levy and J. Marill, Photochem. Photobiol., 2010, 86, 213222.

26 J. Qian, A. Gharibi and S. He, J. Biomed. Opt., 2015, 14, 14012. 27 J. L. Vivero-Escoto and D. L. Vega, RSC Adv., 2014, 4, 14400.
28 L. Latterini and M. Amelia, Langmuir, 2009, 25, 4767-4773. 29 G. Alberto, I. Miletto, G. Viscardi, G. Caputo, L. Latterini, S. Coluccia and G. Martra, J. Phys. Chem. C, 2009, 113, 21048-21053.

30 R. Lindberg, J. Sjoblom and G. Sundholm, Colloids Surf., A, 1995, 99, 79-88.

31 K. Suzuki, K. Ikari and H. Imai, J. Am. Chem. Soc., 2004, 126, 462-463.

32 K. Zhang, L.-L. Xu, J.-G. Jiang, N. Calin, K.-F. Lam, S.-J. Zhang, H.-H. Wu, G.-D. Wu, B. Albela, L. Bonneviot and P. Wu, J. Am. Chem. Soc., 2013, 135, 2427-2430.

33 S. Brunauer, P. H. Emmett and E. Teller, J. Am. Chem. Soc., 1938, 60, 309-319.

34 E. P. Barrett, L. G. Joyner and P. P. Halenda, J. Am. Chem. Soc., 1951, 73, 373-380.

35 A. Jiménez-Banzo, X. Ragàs, P. Kapusta and S. Nonell, Photochem. Photobiol. Sci., 2008, 7, 1003-1010.

36 K. J. Klabunde and R. M. Richards, Nanoscale Materials in Chemistry, John Wiley \& Sons, 2nd edn, 2009.

37 C. Robertson, R. Beanland, S. a. Boden, A. L. Hector, R. J. Kashtiban, J. Sloan, D. C. Smith and A. Walcarius, Phys. Chem. Chem. Phys., 2015, 17, 4763-4770.

38 T. Schiestel, H. Brunner and G. E. M. Tovar, J. Nanosci. Nanotechnol., 2004, 4, 504-511.

39 R. Selvaggi, L. Tarpani, A. Santuari, S. Giovagnoli and L. Latterini, Appl. Catal., B, 2015, 168, 363-369.

40 C. Aggelidou, T. A. Theodossiou and K. Yannakopoulou, Photochem. Photobiol., 2013, 89, 1011-1019.

41 L. Monsù Scolaro, M. Castriciano, A. Romeo, S. Patanè, E. Cefalì and M. Allegrini, J. Phys. Chem. B, 2002, 106, 2453-2459.

42 A. A. Sivash, Z. Masinovský and G. I. Lozovaya, BioSystems, 1991, 25, 131-140.

43 M. A. Jhonsi, A. Kathiravan and R. Renganathan, Spectrochim. Acta, Part A, 2008, 71, 1507-1511.

44 F. Ricchelli, J. Photochem. Photobiol., B, 1995, 29, 109-118.

45 M. A. Filatov, S. Baluschev and K. Landfester, Chem. Soc. Rev., 2016, 45, 4668-4689.

46 S. Oelckers, T. Hanke and B. Roder, J. Photochem. Photobiol., A, 2000, 132, 29-32.

47 K. K. Iu and J. K. Thomas, J. Photochem. Photobiol., A, 1993, 71, 55-60.

48 R. H. Young, R. L. Martin, D. Feriozi, D. Brewer and R. Kayser, Photochem. Photobiol., 1973, 17, 233-244.

49 B. M. Monroe, J. Phys. Chem., 1977, 81, 1861-1864.

50 B. Cojocaru, M. Laferrière, E. Carbonell, V. Parvulescu, H. García and J. C. Scaiano, Langmuir, 2008, 24, 4478-4481. 51 M. Hild and R. Schmidt, J. Phys. Chem. A, 1999, 103, 6091-6096. 52 E. Boix-Garriga, B. Rodríguez-Amigo, O. Planas and S. Nonell, in Singlet Oxygen: Applications in Biosciences and Nanosciences, Royal Society of Chemistry, 2016, vol. 1, pp. 23-46.

53 B. Gottenbos, D. W. Grijpma, H. C. van der Mei, J. Feijen and H. J. Busscher, J. Antimicrob. Chemother., 2001, 48, 7-13.

54 T. J. Silhavy, D. Kahne and S. Walker, Cold Spring Harbor Perspect. Biol., 2010, 2, 1-16.

55 A. M. El Badawy, R. G. Silva, B. Morris, K. G. Scheckel, M. T. Suidan and T. M. Tolaymat, Environ. Sci. Technol., 2011, 45, 283-287. 